\title{
BOUNDED DISTORTION HOMEOMORPHISMS ON ULTRAMETRIC SPACES
}

\author{
Bruce Hughes, Álvaro Martínez-Pérez and Manuel A. Morón \\ Vanderbilt University, Department of Mathematics \\ 1326 Stevenson Center, Nashville, TN 37240, U.S.A.; bruce.hughes@vanderbilt.edu \\ Universidad Complutense de Madrid, Departamento de Geometría y Topología \\ Plaza de Ciencias 3, Madrid 28040, Spain; alvaro_martinez@mat.ucm.es \\ Universidad Complutense de Madrid, Departamento de Geometría y Topología \\ Plaza de Ciencias 3, Madrid 28040, Spain; mamoron@mat.ucm.es
}

Dedicated to José María Montesinos on the occasion of his 65th birthday.

\begin{abstract}
It is well-known that quasi-isometries between $\mathbf{R}$-trees induce power quasi-symmetric homeomorphisms between their ultrametric end spaces. This paper investigates power quasisymmetric homeomorphisms between bounded, complete, uniformly perfect, ultrametric spaces (i.e., those ultrametric spaces arising up to similarity as the end spaces of bushy trees). A bounded distortion property is found that characterizes power quasi-symmetric homeomorphisms between such ultrametric spaces that are also pseudo-doubling. Moreover, examples are given showing the extent to which the power quasi-symmetry of homeomorphisms is not captured by the quasiconformal and bi-Hölder conditions for this class of ultrametric spaces.
\end{abstract}

\section{Introduction}

A theme in the study of noncompact spaces is the extent to which the geometry and topology of such a space is reflected in a natural boundary at infinity.

By choosing a root $v$ for an $\mathbf{R}$-tree $T$, the boundary at infinity naturally becomes a complete, ultrametric space end $(T, v)$ of diameter $\leq 1$, called the end space of $(T, v)$. It is known that a quasi-isometry between $\mathbf{R}$-trees induces a bi-Hölder, quasiconformal homeomorphism between their ultrametric end spaces. In fact, the induced homeomorphism has the stronger power quasi-symmetric, or PQ-symmetric, property.

This paper is concerned with the natural question, How close do the bi-Hölder and quasiconformal conditions come to characterizing PQ-symmetric homeomorphisms on bounded, complete ultrametric spaces? What if one restricts to bounded, complete, uniformly perfect, ultrametric spaces? The significance of restricting to this class of ultrametric spaces is that they are (up to similarity) exactly the ones that arise as end spaces of bushy $\mathbf{R}$-trees. We introduce a notion of bounded distortion for homeomorphisms and a pseudo-doubling property of metric spaces. We show that the bounded distortion property characterizes PQ-symmetric homeomorphisms on

doi:10.5186/aasfm.2010.3529

2000 Mathematics Subject Classification: Primary 54E40, 30C65, 53C23.

Key words: Tree, real tree, bushy tree, ultrametric, end space, quasi-isometry, quasiconformal, quasi-symmetric, PQ-symmetric, doubling metric space.

The first-named author is supported in part by NSF Grant DMS-0504176.

The second- and third-named authors are partially supported by MTM 2006-00825. 
bounded, complete, uniformly perfect, ultrametric spaces that also have the pseudodoubling property. The following is a statement of our main positive result.

Theorem 1.1. A homeomorphism $h: X \rightarrow Y$ between bounded, complete, uniformly perfect, pseudo-doubling, ultrametric spaces is PQ-symmetric if and only if $h$ is a bounded distortion equivalence.

The class of ultrametric spaces to which Theorem 1.1 applies includes end spaces of rooted, geodesically complete, bushy, simplicial R-trees - see Remark 5.10.

The more difficult part of the proof of Theorem 1.1 is in showing that bounded distortion equivalences on the given class of ultrametric spaces are PQ-symmetric. This is accomplished in Corollary 5.11. The proof of that corollary relies on Theorem 5.5, which establishes Theorem1.1 for the case of end spaces of rooted, geodesically complete, simplicial, bushy $\mathbf{R}$-trees. The converse of Theorem 1.1 is Proposition 3.6.

In Example 6.8 it is shown that this theorem does not hold for compact, uniformly perfect, ultrametric spaces that are not pseudo-doubling. Moreover, we give several examples in Section 6 illuminating the difference between bi-Hölder, quasiconformal homeomorphisms on one hand, and PQ-symmetric homeomorphisms on the other hand for this class of ultrametric spaces. In fact, the examples are defined on end spaces of locally finite, simplicial trees of minimal vertex degree three and answer several questions of Mirani [11, 12].

The study of quasi-isometries between trees and the induced maps on their end spaces has a voluminous literature. This is often set in the more general context of hyperbolic metric spaces and their boundaries. See Bonk and Schramm [1], Buyalo and Schroeder [3], Ghys and de la Harpe [5], Martínez-Pérez [9], Mirani [11, 12], and Paulin [14] to name a few. For homeomorphisms induced by $\mathbf{R}$-tree morphisms that are less restrictive than quasi-isometries, see Martínez-Pérez and Morón [10]. For $\mathbf{R}$-tree morphisms more restrictive than quasi-isometries, see Hughes [7].

\section{Preliminaries on trees, end spaces, and ultrametrics}

In this section, we recall the definitions of the trees and their end spaces that are relevant to this paper. We also describe a well-known correspondence between trees and ultrametric spaces. See Feĭnberg [4] for an early result along these lines and Hughes [7] for additional background.

Definition 2.1. Let $(T, d)$ be a metric space.

(1) $(T, d)$ is an $\mathbf{R}$-tree if $T$ is uniquely arcwise connected and for all $x, y \in T$, the unique arc from $x$ to $y$, denoted $[x, y]$, is isometric to the subinterval $[0, d(x, y)]$ of $\mathbf{R}$.

(2) A rooted $\mathbf{R}$-tree $(T, v)$ consists of an $\mathbf{R}$-tree $(T, d)$ and a point $v \in T$, called the root.

(3) A rooted $\mathbf{R}$-tree $(T, v)$ is geodesically complete if every isometric embedding $f:[0, t] \rightarrow T$ with $t>0$ and $f(0)=v$ extends to an isometric embedding $F:[0, \infty) \rightarrow T$.

(4) A simplicial $\mathbf{R}$-tree is an $\mathbf{R}$-tree $(T, d)$ such that $T$ is the (geometric realization of) a simplicial complex and every edge of $T$ is isometric to the closed unit interval $[0,1]$.

Definition 2.2. An ultrametric space is a metric space $(X, d)$ such that $d(x, y) \leq$ $\max \{d(x, z), d(z, y)\}$ for all $x, y, z \in X$. 
Definition 2.3. The end space of a rooted $\mathbf{R}$-tree $(T, v)$ is given by: $\operatorname{end}(T, v)=\{F:[0, \infty) \rightarrow T \mid F(0)=v$ and $F$ is an isometric embedding $\}$. Let $F, G \in \operatorname{end}(T, v)$.

(1) The Gromov product at infinity is $(F \mid G)_{v}:=\sup \{t \geq 0 \mid F(t)=G(t)\}$.

(2) The end space metric is $d_{v}(F, G):=\mathrm{e}^{-(F \mid G)_{v}}$.

(3) The arc $F\left(\left[0,(F \mid G)_{v}\right]\right)$ is denoted $[F \mid G]$.

(4) The bifurcation point of $F$ and $G$ is $F\left((F \mid G)_{v}\right) \in T$.

Proposition 2.4. If $(T, v)$ is a rooted $\mathbf{R}$-tree, then $\left(\operatorname{end}(T, v), d_{v}\right)$ is a complete ultrametric space of diameter $\leq 1$.

Definition 2.5. Let $(U, d)$ be a complete ultrametric space with diameter $\leq 1$. Define an equivalence relation $\sim$ on $U \times[0, \infty)$ by:

$$
(x, t) \sim\left(y, t^{\prime}\right) \Longleftrightarrow t=t^{\prime} \text { and } d(x, y) \leq \mathrm{e}^{-t} .
$$

Then $T_{U}:=U \times[0, \infty) / \sim$ is the tree associated to $(U, d)$. Define a metric $D$ on $T_{U}$ by:

$$
D([x, t],[y, s])= \begin{cases}|t-s| & \text { if } x=y \\ t+s-2 \min \{-\ln (d(x, y)), t, s\} & \text { if } x \neq y\end{cases}
$$

A proof of the first item in the following proposition can be found in [7, Theorem 6.3] and a proof of the second item can be found in [7, Proposition 6.4] and [10, Proposition 5.5].

Proposition 2.6. Let $(U, d)$ be a complete, ultrametric space of diameter $\leq 1$ and let $\left(T_{U}, D\right)$ be its associated tree.

(1) $\left(T_{U}, D\right)$ is a rooted, geodesically complete $\mathbf{R}$-tree with root $v=[x, 0]$ for any $x \in U$.

(2) $U$ is isometric to end $\left(T_{U}, v\right)$.

In this article a map is a function that needs not be continuous.

Definition 2.7. A map $f: X \rightarrow Y$ between metric spaces $\left(X, d_{X}\right)$ and $\left(Y, d_{Y}\right)$ is a quasi-isometric map if there are constants $\lambda \geq 1$ and $A>0$ such that for all $x, x^{\prime} \in X$,

$$
\frac{1}{\lambda} d_{X}\left(x, x^{\prime}\right)-A \leq d_{Y}\left(f(x), f\left(x^{\prime}\right)\right) \leq \lambda d_{X}\left(x, x^{\prime}\right)+A .
$$

If $f(X)$ is a net in $Y$ (i.e., there exists $\epsilon>0$ such that for each $y \in Y$ there exists $x \in X$ such that $\left.d_{Y}(f(x), y)<\epsilon\right)$, then $f$ is a quasi-isometry and $X, Y$ are quasiisometric.

Remark 2.8. It is well-known that a quasi-isometry $f: T \rightarrow T^{\prime}$ between rooted $\mathbf{R}$-trees $(T, v)$ and $\left(T^{\prime}, w\right)$ induces a homeomorphism $\tilde{f}: \operatorname{end}(T, v) \rightarrow \operatorname{end}\left(T^{\prime}, w\right)$. See, for example, Bridson and Haefliger [2, Chapter I.8], where they work in the more general setting of proper, geodesic, metric spaces.

For a quasi-isometry $f: X \rightarrow Y$ between Gromov hyperbolic, almost geodesic metric spaces, Bonk and Schramm define [1, Proposition 6.3] the induced map $\partial f: \partial X \rightarrow \partial Y$ between the boundaries at infinity and prove [1, Theorem 6.5$]$ that $\partial f$ is PQ-symmetric (see Definition 3.4 below) with respect to any metrics on $\partial X$ and $\partial Y$ in their canonical gauges. In the special case that $X$ and $Y$ are $\mathbf{R}$-trees, 
$\partial X=\operatorname{end}(X, v), \partial Y=\operatorname{end}(Y, w)$ and the end space metrics are in the canonical gauges for any choice of roots.

Another source for the result that quasi-isometries between $\mathbf{R}$-trees induce PQsymmetric homeomorphisms on their ultrametric end spaces, is Buyalo and Schroeder [3, Theorem 5.2.17]. They work with Gromov hyperbolic, geodesic metric spaces and with visual boundaries on their boundaries. When specialized to $\mathbf{R}$-trees, these boundaries are the ultrametric end spaces.

\section{Homeomorphisms on metric spaces}

In this section we begin our discussion of various geometric properties that may be satisfied by homeomorphisms between metric spaces. Homeomorphisms between end spaces of rooted, geodesically complete $\mathbf{R}$-trees induced by quasi-isometries of the trees are examples where these properties are encountered. With the possible exceptions of homeomorphisms of bounded distortion and bounded distortion equivalences, defined in Definition 3.2 below, all of these concepts are well-known. In addition to Buyalo and Schroeder [3], other sources for background include Bonk and Schramm [1], Heinonen [6], Roe [15], Semmes [16], and Tukia and Väisälä [17].

Definition 3.1. Let $f: X \rightarrow Y$ be a homeomorphism between metric spaces $\left(X, d_{X}\right)$ and $\left(Y, d_{Y}\right)$. If $x_{0} \in X$ and $\epsilon>0$, then the distortion by $f$ of the $\epsilon$-sphere $S\left(x_{0}, \epsilon\right):=\left\{x \in X \mid d_{X}\left(x_{0}, x\right)=\epsilon\right\}$ at $x_{0}$ is

$$
D_{f}\left(x_{0}, \epsilon\right):=\left\{\begin{array}{cc}
\frac{\sup \left\{d_{Y}\left(f\left(x_{0}\right), f(x)\right) \mid d_{X}\left(x_{0}, x\right)=\epsilon\right\}}{\inf \left\{d_{Y}\left(f\left(x_{0}\right), f(x)\right) \mid d_{X}\left(x_{0}, x\right)=\epsilon\right\}} & \text { if } S\left(x_{0}, \epsilon\right) \neq \emptyset \\
1 & \text { if } S\left(x_{0}, \epsilon\right)=\emptyset .
\end{array}\right.
$$

Definition 3.2. Let $f: X \rightarrow Y$ be a homeomorphism between metric spaces.

(1) $f$ is conformal if $\limsup _{\epsilon \rightarrow 0} D_{f}\left(x_{0}, \epsilon\right)=1$ for all $x_{0} \in X$.

(2) $f$ is $K$-quasiconformal, where $K>0$, if $\limsup _{\epsilon \rightarrow 0} D_{f}(x, \epsilon) \leq K$ for all $x \in X$.

(3) $f$ is quasiconformal if $f$ is $K$-quasiconformal for some $K>0$.

(4) $f$ has bounded distortion if there exists $K>0$ such that

$$
\sup _{x \in X} \sup _{\epsilon>0} D_{f}(x, \epsilon) \leq K
$$

(5) $f$ is a bounded distortion equivalence if $f$ and $f^{-1}: Y \rightarrow X$ have bounded distortion.

Quasiconformal maps are those maps with control on the distortion of sufficiently small spheres. Homeomorphisms of bounded distortion have control on the distortion of every sphere. An example of a quasiconformal (in fact, conformal) homeomorphism that is not of bounded distortion is provided by Example 6.2 below.

Definition 3.3. A map $f: X \rightarrow Y$ between metric spaces $\left(X, d_{X}\right)$ and $\left(Y, d_{Y}\right)$ is quasi-symmetric if $f$ is not constant and if there is a homeomorphism $\eta:[0, \infty) \rightarrow$ $[0, \infty)$, called the control function for $f$, such that whenever $x, a, b \in X, t \geq 0$, and $d_{X}(x, a) \leq t d_{X}(x, b)$ it follows that $d_{Y}(f(x), f(a)) \leq \eta(t) d_{Y}(f(x), f(b))$.

Thus, a quasi-symmetric map controls distortion of annuli; more precisely, it controls the distortion of ratios between inner and outer radii of annuli. 
Definition 3.4. A quasi-symmetric map is said to be power quasi-symmetric, or $P Q$-symmetric, if it has a control function of the form

$$
\eta(t)=q \max \left\{t^{p}, t^{1 / p}\right\}
$$

for some $p, q \geq 1$.

Remark 3.5. It follows from Tukia and Väisälä [17, Theorem 2.2] that inverses and compositions of quasi-symmetric homeomorphisms are quasi-symmetric; moreover, inverses and compositions of PQ-symmetric homeomorphisms are PQsymmetric.

Proposition 3.6. If a homeomorphism $f: X \rightarrow Y$ between metric spaces $\left(X, d_{X}\right)$ and $\left(Y, d_{Y}\right)$ is quasi-symmetric, then $f$ is a bounded distortion equivalence.

Proof. Let $\eta$ be a control function for $f$ and let $x \in X$ and $\epsilon>0$ be given. If $a, b \in X$ with $d_{X}(x, a)=\epsilon=d_{X}(x, b)$, then $d_{Y}(f(x), f(a)) \leq \eta(1) d_{Y}(f(x), f(b))$. Hence, $D_{f}(x, \epsilon) \leq \eta(1)$ and $f$ has bounded distortion with constant $K=\eta(1)$. It follows from Remark 3.5 that $f^{-1}$ is also quasi-symmetric (hence, of bounded distortion) and $f$ is a bounded distortion equivalence.

Definition 3.7. A homeomorphism $f: X \rightarrow Y$ between metric spaces $\left(X, d_{X}\right)$ and $\left(Y, d_{Y}\right)$ is bi-Hölder if there exists constants $\alpha>0$ and $c>0$ such that

$$
\frac{1}{c} d_{X}(x, y)^{1 / \alpha} \leq d_{Y}(f(x), f(y)) \leq c d_{X}(x, y)^{\alpha}
$$

for all $x, y \in X$. If $\alpha=1$, then $f$ is bi-Lipschitz.

Remark 3.8. Note that a bi-Lipschitz homeomorphism is PQ-symmetric with $p=1, q=c^{2}$, and $\eta(t)=q t$. It follows from the proof of Tukia and Väisälä [17, Theorem 3.14] that a PQ-symmetric homeomorphism $f: X \rightarrow Y$ between bounded metric spaces is bi-Hölder. A quasiconformal homeomorphism need not be bi-Hölder as shown in Example 6.3 below.

Remark 3.9. For homeomorphisms between arbitrary bounded metric spaces, there are the following implications:

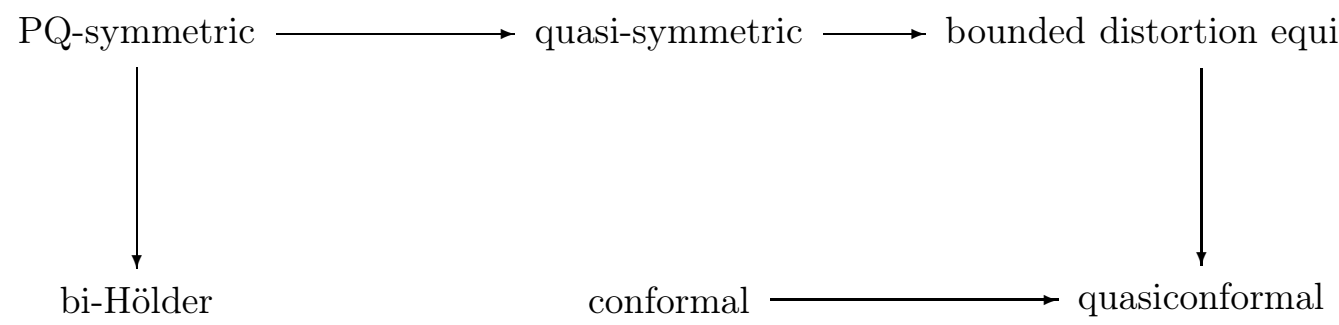

In fact, the implication that PQ-symmetric homeomorphisms are bi-Hölder is the only one that requires that the metric spaces be bounded. No other implications hold in general.

\section{Homeomorphisms on ultrametric spaces}

In this section, the Gromov product at infinity is used to characterize homeomorphisms of bounded distortion, quasi-symmetries, and PQ-symmetries in the case of interest to us, namely, maps between end spaces of trees. It is also shown that local similarity equivalences between compact, ultrametric spaces are PQ-symmetric. 
We begin with the characterization of bounded distortion homeomorphisms in terms of the Gromov product at infinity.

Proposition 4.1. If $(T, v)$ and $\left(T^{\prime}, w\right)$ are rooted, geodesically complete $\mathbf{R}$ trees, then a homeomorphism $h: \operatorname{end}(T, v) \rightarrow \operatorname{end}\left(T^{\prime}, w\right)$ has bounded distortion if and only if there exists a constant $A \geq 0$ such that whenever $F, G, H \in \operatorname{end}(T, v)$ and $(F \mid G)_{v}=(F \mid H)_{v}$ it follows that $\left|(h(F) \mid h(G))_{w}-(h(F) \mid h(H))_{w}\right| \leq A$.

Proof. Using the relation between the end space metric and the Gromov product at infinity, it follows that for $F \in \operatorname{end}(T, v), \epsilon>0$, and $K \geq 1$, we have $D_{h}(F, \epsilon) \leq K$ if and only if

$$
\frac{\mathrm{e}^{-(h(F) \mid h(G))_{w}}}{\mathrm{e}^{-(h(F) \mid h(H))_{w}}} \leq K,
$$

whenever $G, H \in \operatorname{end}(T, v)$ and $(F \mid G)_{v}=(F \mid H)_{v}=-\ln \epsilon$. Thus, if $F \in \operatorname{end}(T, v)$ and $K \geq 1$, then $\sup _{\epsilon>0} D_{h}(F, \epsilon) \leq K$ if and only if

$$
\mathrm{e}^{(h(F) \mid h(H))_{w}-(h(F) \mid h(G))_{w}} \leq K,
$$

whenever $G, H \in \operatorname{end}(T, v)$ and $(F \mid G)_{v}=(F \mid H)_{v}$; in turn, this holds if and only if

$$
\left|(h(F) \mid h(H))_{w}-(h(F) \mid h(G))_{w}\right| \leq \ln K,
$$

whenever $G, H \in \operatorname{end}(T, v)$ and $(F \mid G)_{v}=(F \mid H)_{v}$. The result follows; furthermore, the relationship between the bounded distortion constant $K$ and the constant $A$ is given by $A=\ln K$.

The following result gives the characterization of quasi-symmetric maps between end spaces of trees in terms of the Gromov product at infinity.

Proposition 4.2. If $(T, v)$ and $\left(T^{\prime}, w\right)$ are rooted, geodesically complete $\mathbf{R}$ trees, then a map $f: \operatorname{end}(T, v) \rightarrow \operatorname{end}\left(T^{\prime}, w\right)$ is quasi-symmetric if and only if there exists an orientation-preserving homeomorphism $\gamma: \mathbf{R} \rightarrow \mathbf{R}$ such that whenever $F, G, H \in \operatorname{end}(T, v)$, it follows that

$$
-\gamma\left((F \mid G)_{v}-(F \mid H)_{v}\right) \leq\left(F^{\prime} \mid H^{\prime}\right)_{w}-\left(F^{\prime} \mid G^{\prime}\right)_{w} \leq \gamma\left((F \mid H)_{v}-(F \mid G)_{v}\right),
$$

where $F^{\prime}=f(F), G^{\prime}=f(G)$, and $H^{\prime}=f(H)$.

Proof. According to Tukia and Väisälä [17, page 99], $f$ is quasi-symmetric if and only if there exists a homeomorphism $\eta:[0, \infty) \rightarrow[0, \infty)$ such that

$$
\eta\left(\rho^{-1}\right)^{-1} \leq \frac{d_{w}\left(F^{\prime}, G^{\prime}\right)}{d_{w}\left(F^{\prime}, H^{\prime}\right)} \leq \eta(\rho)
$$

whenever $F, G, H \in \operatorname{end}(T, v)$ and $\rho=\frac{d_{v}(F, G)}{d_{v}(F, H)}$. The result now follows easily via the correspondence of $\eta$ and $\gamma$ implied by the relation $\gamma(t)=\ln \eta\left(\mathrm{e}^{t}\right)$ and the relation between the end space metric and the Gromov product at infinity.

The following result gives the characterization of PQ-symmetric maps between end spaces of trees in terms of the Gromov product at infinity.

Proposition 4.3. If $(T, v)$ and $\left(T^{\prime}, w\right)$ are rooted, geodesically complete $\mathbf{R}$ trees, then a map $f: \operatorname{end}(T, v) \rightarrow \operatorname{end}\left(T^{\prime}, w\right)$ is $P Q$-symmetric if and only if there exist constants $\lambda \geq 1$ and $A \geq 0$ such that whenever $F, G, H \in \operatorname{end}(T, v)$ and 
$(F \mid G)_{v} \leq(F \mid H)_{v}<\infty$, it follows that

$$
\frac{1}{\lambda}\left((F \mid H)_{v}-(F \mid G)_{v}\right)-A \leq\left(F^{\prime} \mid H^{\prime}\right)_{w}-\left(F^{\prime} \mid G^{\prime}\right)_{w} \leq \lambda\left((F \mid H)_{v}-(F \mid G)_{v}\right)+A,
$$

where $F^{\prime}=f(F), G^{\prime}=f(G)$ and $H^{\prime}=f(H)$.

Proof. Suppose first that $f$ is PQ-symmetric with constants $p, q \geq 1$ as in Definition 3.4 and let $\lambda=p$ and $A=\ln q$. If $F, G, H \in \operatorname{end}(T, v)$ and $(F \mid G)_{v} \leq$ $(F \mid H)_{v}<\infty$, then it follows from the relation between the end space metric and the Gromov product at infinity that

$$
\frac{d_{v}(F, G)}{d_{v}(F, H)}=\mathrm{e}^{(F \mid H)_{v}-(F \mid G)_{v}} \geq 1 .
$$

Thus, $d_{v}(F, G)=t \cdot d_{v}(F, H)$, where $t \geq 1$ and $\ln t=(F \mid H)_{v}-(F \mid G)_{v}$. The PQsymmetric property implies

$$
d_{w}\left(F^{\prime}, G^{\prime}\right) \leq q t^{p} d_{w}\left(F^{\prime}, H^{\prime}\right)
$$

and

$$
d_{w}\left(F^{\prime}, H^{\prime}\right) \leq q(1 / t)^{1 / p} d_{w}\left(F^{\prime}, G^{\prime}\right) .
$$

These two inequalities are equivalent to

$$
\left(F^{\prime} \mid G^{\prime}\right)_{w} \geq\left(F^{\prime} \mid H^{\prime}\right)_{w}-\lambda\left((F \mid H)_{v}-(F \mid G)_{v}\right)-A
$$

and

$$
\left(F^{\prime} \mid H^{\prime}\right)_{w} \geq\left(F^{\prime} \mid G^{\prime}\right)_{w}+\frac{1}{\lambda}\left((F \mid H)_{v}-(F \mid G)_{v}\right)-A,
$$

which are in turn equivalent to the desired inequalities.

Conversely, given $\lambda \geq 1$ and $A \geq 0$ satisfying the given conditions, let $p=\lambda$ and $q=\mathrm{e}^{A}$. Suppose $F, G, H \in \operatorname{end}(T, v), t \geq 0$, and $d_{v}(F, G) \leq t d_{v}(F, H)$. It must be shown that

$$
d_{w}\left(F^{\prime}, G^{\prime}\right) \leq \begin{cases}q t^{p} d_{w}\left(F^{\prime}, H^{\prime}\right) & \text { if } t \geq 1 \\ q t^{1 / p} d_{w}\left(F^{\prime}, H^{\prime}\right) & \text { if } t \leq 1\end{cases}
$$

(where $F^{\prime}, G^{\prime}, H^{\prime}$ continue to denote the images of $F, G, H$, respectively, under $f$ ). We may assume $0<d_{v}(F, G)$ and $t>0$, for otherwise the result is trivial.

We first consider the case $(F \mid G)_{v} \leq(F \mid H)_{v}$. In particular, $d_{v}(F, H) \leq d_{v}(F, G)$ and $t \geq 1$. The right-hand part of the assumed inequalities takes the form

$$
\ln \frac{d_{w}\left(F^{\prime}, G^{\prime}\right)}{d_{w}\left(F^{\prime}, H^{\prime}\right)} \leq p \ln \frac{d_{v}(F, G)}{d_{v}(F, H)}+\ln q=\ln \left[q\left(\frac{d_{v}(F, G)}{d_{v}(F, H)}\right)^{p}\right] \text {. }
$$

Therefore,

$$
\frac{d_{w}\left(F^{\prime}, G^{\prime}\right)}{d_{w}\left(F^{\prime}, H^{\prime}\right)} \leq q\left(\frac{d_{v}(F, G)}{d_{v}(F, H)}\right)^{p} \leq q t^{p}
$$

as required.

Finally, consider the case $(F \mid H)_{v} \leq(F \mid G)_{v}$. The left-hand part of the assumed inequalities takes the form

$$
\ln \left[\frac{1}{q}\left(\frac{d_{v}(F, H)}{d_{v}(F, G)}\right)^{1 / p}\right]=\frac{1}{p} \ln \frac{d_{v}(F, H)}{d_{v}(F, G)}-\ln q \leq \ln \frac{d_{w}\left(F^{\prime}, H^{\prime}\right)}{d_{w}\left(F^{\prime}, G^{\prime}\right)} .
$$


Therefore,

$$
\frac{1}{q}\left(\frac{1}{t}\right)^{1 / p} \leq \frac{1}{q}\left(\frac{d_{v}(F, H)}{d_{v}(F, G)}\right)^{1 / p} \leq \frac{d_{w}\left(F^{\prime}, H^{\prime}\right)}{d_{w}\left(F^{\prime}, G^{\prime}\right)}
$$

and

$$
d_{w}\left(F^{\prime}, G^{\prime}\right) \leq q t^{1 / p} d_{w}\left(F^{\prime}, H^{\prime}\right) .
$$

This is exactly what is required if $t \leq 1$; if $t \geq 1$, what is required follows by using $t^{1 / p} \leq t^{p}$

Remark 4.4. The following characterization of bi-Hölderness in terms of the Gromov product at infinity is straightforward to verify. If $(T, v)$ and $\left(T^{\prime}, w\right)$ are rooted, geodesically complete $\mathbf{R}$-trees, then a homeomorphism $f: \operatorname{end}(T, v) \rightarrow \operatorname{end}\left(T^{\prime}, w\right)$ is bi-Hölder if and only if there exist constants $\lambda \geq 1$ and $A \geq 0$ such that whenever $F, G \in \operatorname{end}(T, v)$, it follows that

$$
\frac{1}{\lambda}(F \mid G)_{v}-A \leq\left(F^{\prime} \mid G^{\prime}\right)_{w} \leq \lambda(F \mid G)_{v}+A
$$

where $F^{\prime}=f(F)$ and $G^{\prime}=f(G)$.

Remark 4.5. Suppose $(T, v)$ and $\left(T^{\prime}, w\right)$ are rooted, geodesically complete, Rtrees and $h: \operatorname{end}(T, v) \rightarrow \operatorname{end}\left(T^{\prime}, w\right)$ is a homeomorphism induced by a rooted homeomorphism $\hat{h}:(T, v) \rightarrow\left(T^{\prime}, w\right)$; i.e., $h(F)=\hat{h} \circ F$ for all $F \in \operatorname{end}(T, v)$. It follows that if $F, G, H \in \operatorname{end}(T, v)$ and $(F \mid G)_{v}=(F \mid H)_{v}$, then $(h(F) \mid h(G))_{w}=(h(F) \mid h(H))_{w}$. Thus, $h$ is conformal and a bounded distortion equivalence (with constant $K=1$ ).

Finally, we show that the local similarity equivalences between compact ultrametric spaces studied in $[7,8]$ are PQ-symmetric. In fact, we show that they are bi-Lipschitz. In particular, this affirms a conjecture of Mirani [11, 12].

Definition 4.6. A function $f: X \rightarrow Y$ between metric spaces $\left(X, d_{X}\right),\left(Y, d_{Y}\right)$ is a similarity if there exists $\lambda>0$ such that $d_{Y}(f(x), f(y))=\lambda d_{X}(x, y)$ for all $x, y \in X$. In this case, $f$ is a $\lambda$-similarity.

Definition 4.7. A homeomorphism $h: X \rightarrow Y$ between metric spaces is a local similarity equivalence if for every $x \in X$ there exist $\varepsilon>0$ and $\lambda>0$ such that the restriction $\left.h\right|_{B(x, \varepsilon)}: B(x, \varepsilon) \rightarrow B(h(x), \lambda \varepsilon)$ is a surjective $\lambda$-similarity.

Proposition 4.8. If $f: U \rightarrow V$ is a local similarity equivalence between compact, ultrametric spaces, then $f$ is bi-Lipschitz. In particular, $f$ is $P Q$-symmetric.

Proof. Up to similarity homeomorphism, we may assume that the diameters of $U$ and $V$ are $\leq 1$. Proposition 2.6 then implies that $U=\operatorname{end}(T, v)$ and $V=$ end $\left(T^{\prime}, w\right)$, where $(T, v)$ and $\left(T^{\prime}, w\right)$ are rooted, geodesically complete $\mathbf{R}$-trees. There is a local similarity equivalence $h: \operatorname{end}(T, v) \rightarrow \operatorname{end}\left(T^{\prime}, w\right)$ induced by conjugating $f$ by similarities. Clearly, it suffices to show that $h$ is bi-Lipschitz. To this end, we will show that there exists some constant $K>0$ such that if $F, G \in \operatorname{end}(T, v)$, then $\left|(F \mid G)_{v}-(h(F) \mid h(G))_{w}\right| \leq K$. If there is such a constant $K$, then it is clear that: if $F, G \in \operatorname{end}(T, v)$, then $(F \mid G)_{v}-K \leq(h(F) \mid h(G))_{w} \leq(F \mid G)_{v}+K$ and $e^{-K} d(F, G) \leq d(h(F), h(G)) \leq e^{K} d(F, G)$. That is, $h$ is bi-Lipschitz.

To complete the proof, we will establish the existence of $K$. For every $F \in$ $\operatorname{end}(T, v)$ there exist $\varepsilon>0$ and $\lambda>0$ such that the restriction $\left.h\right|_{B(x, \varepsilon)}: B(x, \varepsilon) \rightarrow$ $B(h(x), \lambda \varepsilon)$ is a surjective $\lambda$-similarity. Since end $(T, v)$ is compact, there is a finite, 
open covering $\left\{B\left(x_{i}, \varepsilon_{i}\right)\right\}_{i=1}^{n}$ and associated similarity constants $\left\{\lambda_{i}\right\}_{i=1}^{n}$. Since $h$ is a homeomorphism, $\left.\left\{B\left(h\left(x_{i}\right), \lambda_{i} \varepsilon_{i}\right)\right)\right\}_{i=1}^{n}$ is also an open covering of end $\left(T^{\prime}, w\right)$. Let $\delta_{1}$ a Lebesgue number for the covering $\left\{B\left(x_{i}, \varepsilon_{i}\right)\right\}_{i=1}^{n}$ and $\delta_{2}$ a Lebesgue number for the covering $\left\{B\left(h\left(x_{i}\right), \lambda_{i} \varepsilon_{i}\right)\right\}_{i=1}^{n}$. Define

$$
K:=\max \left\{\max \left\{\left|\ln \left(\lambda_{i}\right)\right| \mid i=1, \ldots, n\right\},-2 \ln \left(\delta_{1}\right),-2 \ln \left(\delta_{2}\right)\right\} .
$$

If $F, G \in B\left(x_{i}, \varepsilon_{i}\right)$ for some $i=1, \ldots, n$, then $d(h(F), h(G))=\lambda_{i} d(F, G)$ and $\left|(F \mid G)_{v}-(h(F) \mid h(G))_{w}\right|=\left|\ln \left(\lambda_{i}\right)\right| \leq K$, as desired. On the other hand, if $F, G$ are not in any such ball, then $h(F)$ and $h(G)$ are not in the same ball $\left.B\left(h\left(x_{i}\right), \lambda_{i} \varepsilon_{i}\right)\right)$ for any $i$; therefore, $d(h(F), h(G))>\delta_{2}$. Thus, $\left|(F \mid G)_{v}-(h(F) \mid h(G))_{w}\right| \leq(F \mid G)_{v}+$ $(h(F) \mid h(G))_{w} \leq-\ln \left(\delta_{1}\right)-\ln \left(\delta_{2}\right) \leq K$, which establishes the desired property of $K$.

Remark 4.9. An alternative proof that a local similarity equivalence between compact, ultrametric spaces is PQ-symmetric can be obtained as follows. First, it is possible to modify the proof of Tukia and Väisälä [17, Theorem 2.23] to show that a local PQ-symmetric embedding $f: X \rightarrow Y$ between metric spaces, where $X$ is compact, is PQ-symmetric. Then observe that, since a similarity is PQ-symmetric (with $p=1=q$ ), a local similarity equivalence is locally PQ-symmetric.

\section{Homeomorphisms on uniformly perfect, ultrametric spaces}

In this section, it is shown that a bounded distortion homeomorphism between bounded, complete, uniformly perfect, pseudo-doubling, ultrametric spaces is PQsymmetric - see Corollary 5.11. This is the remaining part of the main result Theorem 1.1, namely, the sufficiency of bounded distortion for PQ-symmetry. The proof of Corollary 5.11 consists of a reduction to the end spaces of simplicial, bushy $\mathbf{R}$ trees. That reduction is contained in Theorem 5.5. This section also contains the definition of pseudo-doubling metric spaces and a brief discussion of its relationship to the well-known notion of doubling metric spaces.

We begin by recalling the following definition of uniformly perfect for metric spaces and its relation to bushy $\mathbf{R}$-trees.

Definition 5.1. A metric space $X$ is uniformly perfect if there is a constant $\mu \in(0,1)$ such that for every $x \in X$ and every $r>0$, it follows that $B_{r}(x) \backslash B_{\mu r}(x) \neq \emptyset$ unless $X=B_{r}(x)$.

Tukia and Väisälä [17] proved that a quasi-symmetric homeomorphism between uniformly perfect metric spaces is PQ-symmetric (see also [6, Theorem 11.3, page 89]).

Now recall the following definition from Mosher, Sageev, and Whyte [13].

Definition 5.2. An R-tree $T$ is bushy if there is a constant $K>0$, called a bushy constant, such that for any point $x \in T$ there is a point $y \in T$ such that $d(x, y)<K$ and $T \backslash\{y\}$ has at least 3 unbounded components.

Remark 5.3. Note that a rooted, geodesically complete $\mathbf{R}$-tree $T$ is bushy if and only if end $(T, v)$ is uniformly perfect for some (respectively, for every) $v \in T$.

Remark 5.4. As Mosher, Sageev, and Whyte [13, page 118] point out, any two bounded valence, locally finite, simplicial, bushy trees are quasi-isometric. Therefore, any such tree is quasi-isometric to the infinite binary tree. See also Bridson and 
Haefliger [2, page 141, Exercise 8.20(2)] for the special case of regular, simplicial trees.

Theorem 5.5. If a map $h: \operatorname{end}(T, v) \rightarrow \operatorname{end}\left(T^{\prime}, w\right)$ between the end spaces of rooted, geodesically complete, simplicial, bushy $\mathbf{R}$-trees is a bounded distortion equivalence, then $h$ is $P Q$-symmetric.

Proof. Let $K>0$ be a bushy constant for both $T$ and $T^{\prime}$, and let $\mathrm{e}^{A}$ be the constant for the bounded distortion equivalence, where $A \geq 0$. Let $p=A+2 K$, $q_{1}=4 K+3 A, q_{2}=\max \left\{q_{1}^{1 / p}, q_{1} \mathrm{e}^{2 A}\right\}$, and $q=\max \left\{q_{1}, q_{2}\right\}$. We will show that $h$ is PQ-symmetric with constants $p$ and $q$.

Consider any three points $G_{0}, G_{1}, G_{2} \in \operatorname{end}(T, v)$ and suppose $d\left(G_{0}, G_{1}\right)=$ $d\left(G_{1}, G_{2}\right) \geq d\left(G_{0}, G_{2}\right)$. Let $t_{0}=\left(G_{0} \mid G_{1}\right)_{v}$ and $t_{1}=\left(G_{0} \mid G_{2}\right)_{v}$. Then, $d\left(G_{0}, G_{1}\right)=$ $t \cdot d\left(G_{0}, G_{2}\right)$ with $t=\mathrm{e}^{t_{1}-t_{0}} \geq 1$. Since $T$ is a simplicial $\mathbf{R}$-tree, there exists $k \in \mathbf{N}$ and vertices $x_{0}, \ldots, x_{k+1} \in T$ as shown in Figure 1 such that

(1) $\left[G_{0} \mid G_{1}\right]=\left[v, x_{0}\right]$ and $\left[G_{0} \mid G_{2}\right]=\left[v, x_{k+1}\right]$

(2) $d\left(x_{0}, x_{k+1}\right)=k+1$

(3) $d\left(v, x_{i}\right)=d\left(v, x_{0}\right)+i \in \mathbf{N}$ for each $i=0, \ldots, k+1$

(4) $x_{i} \in\left[v, x_{k+1}\right]$ for each $i=0, \ldots, k+1$

For each $i=0, \ldots, k+1$, let $\mathscr{F}_{i}=\left\{F \in \operatorname{end}(T, v) \mid\left[F \mid G_{0}\right]=\left[v, x_{i}\right]\right\}$. Note that $\mathscr{F}_{i}$ may be empty.
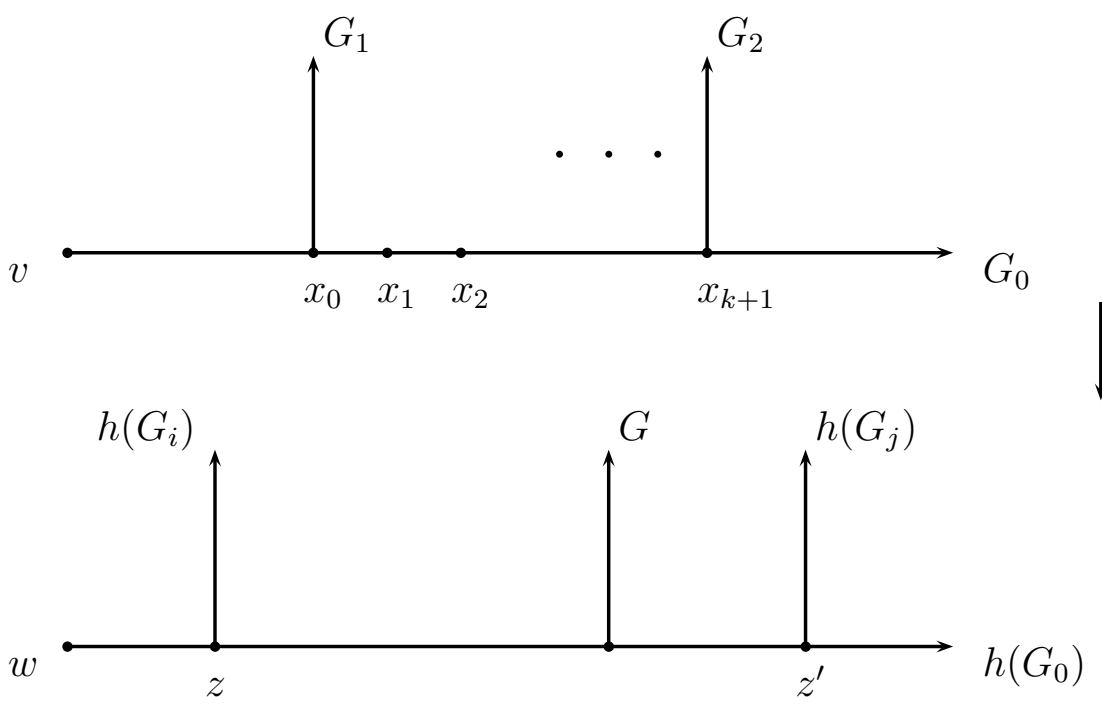

Figure 1. $h: \operatorname{end}(T, v) \rightarrow \operatorname{end}\left(T^{\prime}, w\right)$.

The first step is to show that

$$
d\left(h\left(G_{0}\right), h\left(G_{1}\right) \leq q_{1} \cdot t^{p} d\left(h\left(G_{0}\right), h\left(G_{2}\right)\right) .\right.
$$

Suppose on the contrary that

$$
d\left(h\left(G_{0}\right), h\left(G_{1}\right)>(4 K+3 A) \cdot t^{A+2 K} d\left(h\left(G_{0}\right), h\left(G_{2}\right)\right),\right.
$$

which is to say,

$$
\left(h\left(G_{0}\right) \mid h\left(G_{1}\right)\right)_{w}-\left(h\left(G_{0}\right) \mid h\left(G_{2}\right)\right)_{w}>(k+1)(A+2 K)+4 K+3 A .
$$


For any two $F_{1}, F_{2} \in \mathscr{F}_{i}, 0 \leq i \leq k+1$, the bounded distortion condition with respect to $G_{0}$ implies that

$$
\left|\left(h\left(G_{0}\right) \mid h\left(F_{1}\right)\right)_{w}-\left(h\left(G_{0}\right) \mid h\left(F_{1}\right)\right)_{w}\right| \leq A .
$$

It follows that for each $i=0, \ldots, k+1$, there exists a subinterval $I_{i}$ of $h\left(G_{0}\right)$ of length $A$ such that the bifurcation points of $h\left(G_{0}\right)$ and elements of $h\left(\mathscr{F}_{i}\right)$ and are all contained in the subinterval $I_{i}$. Since $T^{\prime}$ is bushy with constant $K$, there exists $G \in \operatorname{end}\left(T^{\prime}, w\right) \backslash \bigcup_{i=0}^{k+1} h\left(\mathscr{F}_{i}\right)$ such that

$$
\left(h\left(G_{0}\right) \mid h\left(G_{1}\right)\right)_{w}+A<\left(G \mid h\left(G_{0}\right)\right)_{w}<\left(h\left(G_{0}\right) \mid h\left(G_{2}\right)\right)_{w}-A .
$$

Suppose $z$ is the bifurcation point of $h\left(G_{0}\right)$ and $h\left(G_{1}\right)$, and $z^{\prime}$ the bifurcation point of $h\left(G_{0}\right)$ and $h\left(G_{2}\right)$. The interval $I:=\left[G_{0}(\|z\|+A), G_{0}\left(\left\|z^{\prime}\right\|-A\right)\right]$ has length at least $(k+1)(A+2 K)+4 K+A$. Thus, $I$ contains an open interval of length $2 K$ disjoint from each of the $k+2$ subintervals $I_{i}$, for $0 \leq i \leq k+1$. Since $K$ is the bushy constant, there exists $H \in \operatorname{end}\left(T^{\prime}, w\right)$ such that the bifurcation point of $h\left(G_{0}\right)$ and $H$ is in $I$. The surjectivity of $h$ implies there exists $G \in \operatorname{end}(T, v) \backslash \bigcup_{i=0}^{k+1} \mathscr{F}_{i}$ such that $h(G)=H$. There are two cases:

(a) $\left(G \mid G_{0}\right)<\left\|x_{0}\right\|$. This leads to a contradiction by applying the bounded distortion condition on $G$ with respect to $G_{0}, G_{1}$ in conjunction with the fact that $\left|\left(h(G) \mid h\left(G_{0}\right)\right)_{w}-\left(h(G) \mid h\left(G_{1}\right)\right)_{w}\right|>A$.

(b) $\left(G \mid G_{0}\right)>\left\|x_{k+1}\right\|$. This leads to a contradiction by applying the bounded distortion condition on $G_{2}$ with respect to $G, G_{0}$ in conjunction with the fact that $\left(H \mid h\left(G_{0}\right)\right)_{w}<\left(h\left(G_{0}\right) \mid h\left(G_{2}\right)\right)_{w}-A$.

In either case, there is a contradiction. The conclusion is that $d\left(h\left(G_{0}\right), h\left(G_{1}\right)\right) \leq$ $q_{1} t^{p} \cdot d\left(h\left(G_{0}\right), h\left(G_{2}\right)\right)$ and the first step is complete.

The second and final step is to show that

$$
d\left(h\left(G_{0}\right), h\left(G_{2}\right)\right) \leq q_{2}\left(\frac{1}{t}\right)^{\frac{1}{p}} \cdot d\left(h\left(G_{0}\right), h\left(G_{1}\right)\right),
$$

which is to say,

$$
d\left(h\left(G_{0}\right), h\left(G_{1}\right)\right) \geq \frac{1}{q_{2}} t^{\frac{1}{p}} \cdot d\left(h\left(G_{0}\right), h\left(G_{2}\right)\right) .
$$

To this end note that the bounded distortion assumption together with the fact that $d\left(G_{0}, G_{1}\right)=d\left(G_{1}, G_{2}\right) \geq d\left(G_{0}, G_{2}\right)$, implies that

$$
d\left(h\left(G_{0}\right), h\left(G_{1}\right)\right) \geq \mathrm{e}^{-A} \cdot d\left(h\left(G_{0}\right), h\left(G_{2}\right)\right) .
$$

Let $t_{0}^{\prime}=\left(h\left(G_{0}\right) \mid h\left(G_{1}\right)\right)_{v}, t_{1}^{\prime}=\left(h\left(G_{0}\right) \mid h\left(G_{2}\right)\right)_{v}$ and $t^{\prime}=\mathrm{e}^{\left|t_{1}-t_{0}\right|}$. By the same argument as before, $(4 K+3 A) t^{\prime p} \geq t$. Therefore, $t^{\prime} \geq\left(\frac{t}{4 K+3 A}\right)^{\frac{1}{p}}$. In other words, either

$$
d\left(h\left(G_{0}\right), h\left(G_{1}\right)\right) \geq\left(\frac{1}{4 K+3 A}\right)^{\frac{1}{p}} t^{\frac{1}{p}} d\left(h\left(G_{0}\right), h\left(G_{2}\right)\right)
$$

or

$$
\mathrm{e}^{A} d\left(h\left(G_{0}\right), h\left(G_{1}\right)\right) \geq d\left(h\left(G_{0}\right), h\left(G_{2}\right)\right) \geq\left(\frac{1}{4 K+3 A}\right)^{\frac{1}{p}} t^{\frac{1}{p}} d\left(h\left(G_{0}\right), h\left(G_{1}\right)\right),
$$


where the second is only possible if $t^{\frac{1}{p}} \leq(4 K+3 A) \mathrm{e}^{A}$. In the first case, the desired result follows from the fact that $q_{2} \geq(4 K+3 A)^{\frac{1}{p}}$. In the second case,

$$
d\left(h\left(G_{0}\right), h\left(G_{1}\right)\right) \geq \mathrm{e}^{-A} d\left(h\left(G_{0}\right), h\left(G_{2}\right)\right) \geq \mathrm{e}^{-A} \frac{t^{\frac{1}{p}}}{(4 K+3 A) \mathrm{e}^{A}} d\left(h\left(G_{0}\right), h\left(G_{2}\right)\right) .
$$

Thus, the desired result follows from the fact that $q_{2} \geq(4 K+3 A) \mathrm{e}^{2 A}$. In both cases, it is readily seen that $d\left(h\left(G_{0}\right), h\left(G_{1}\right)\right) \geq \frac{1}{q_{2}} t^{\frac{1}{p}} d\left(h\left(G_{0}\right), h\left(G_{2}\right)\right)$, completing the second step. Thus, $h$ is PQ-symmetric.

Definition 5.6. A map between metric spaces $f: X \rightarrow Y$ is roughly isometric if there is a constant $a>0$ such that $d\left(x, x^{\prime}\right)-a \leq d\left(f(x) f\left(x^{\prime}\right)\right) \leq d\left(x, x^{\prime}\right)+a$ for all $x, x^{\prime} \in X$. If, in addition, $f(X)$ is a net in $Y$, then $f$ is a rough isometry and $X$ and $Y$ are roughly isometric.

Obviously, a rough isometry is a quasi-isometry.

Proposition 5.7. If $T$ is an $\mathbf{R}$-tree $T$, then there exists a simplicial $\mathbf{R}$-tree $S$ such that $T$ and $S$ are (continuously) rough isometric. In particular, $T$ and $S$ are (continuously) quasi-isometric.

Proof. Fix a root $v \in T$ and consider $(T, v)$ the rooted R-tree. For each $n=$ $1,2,3, \ldots$, let $S_{n}=\partial B(v, n)$. Let $S_{0}=\{w\}$, where $w$ is a point not in $\bigcup_{i=1}^{\infty} S_{n}$. Define the rooted, simplicial R-tree $(S, w)$ by joining with edges of length 1 all the points in $S_{1}$ to $w$ and each point $s \in S_{k}$ to the unique point $t \in S_{k-1}$ such that $t \in[v, s]$. Thus, $\bigcup_{i=0}^{\infty} S_{i}$ is the vertex set of $S$.

Any isometric embedding of the form $F:[0, \infty) \rightarrow T$ or $F:[0, N] \rightarrow T$, where $F(0)=v$ and $N \in \mathbf{N}$, is completely determined by the sequence $\{F(k)\}_{k=1}^{\infty}$ or $\{F(k)\}_{k=1}^{N}$, respectively. Since these sequences are in the vertex set of $S$, there is a corresponding isometric embedding of the form $F^{\prime}:[0, \infty) \rightarrow S$ or $F^{\prime}:[0, N] \rightarrow S$, where $F^{\prime}(0)=w$. Extend this construction to isometric embeddings of the form $F:[0, t] \rightarrow T$, where $F(0)=v$ and $t>0$, by letting $F^{\prime}$ denote $(F \mid[0,\lfloor t]])^{\prime}$, where $\lfloor t\rfloor$ denotes the greatest integer less than or equal to $t$. Denote this association by $p: F \mapsto F^{\prime}$. (Note that $p$ is surjective between the sets of isometric embeddings. However, $p$ need not be injective because of the existence of domains of the form $[0, t]$ for non-integral t.) Define the map $h:(T, v) \rightarrow(S, w)$ as follows: First, $h(x)=w$ for all $x \in B(v, 1)$. Second, $h(F(t))=F^{\prime}(t-1)$ for any isometric embedding of the form $F:[0, \infty) \rightarrow T$ or $F:[0, t] \rightarrow T$, where $F(0)=v$ and $t \geq 1$. In particular, for every integer $k>0$ in the domain of $F, h(F(k))=F^{\prime}(k-1)$ which, by the definition of $S$, is the point $F(k-1)$. In order to show that $h$ is well-defined, let $F$ and $G$ be two isometric embeddings into $T$ of the type considered above. If $t$ is in the domains of $F$ and $G$, then $F(t)=G(t)$ implies that $F(\lfloor t\rfloor)=G(\lfloor t\rfloor)$. In particular, $F^{\prime}(t-1)=G^{\prime}(t-1)$. Therefore, $h(F(t))=F^{\prime}(t-1)=G^{\prime}(t-1)=h(G(t))$.

Finally, define $j:(S, w) \rightarrow(T, v)$ by $j(H(t))=F(t)$, where $H:[0, \infty) \rightarrow S$ or $H:[0, N] \rightarrow S$ is an isometric embedding such that $H(0)=w$ and $N \in \mathbf{N}$, and $F$ is an isometric embedding in $T$ such that $p(F)=H$. Note that $j(H(t))$ does not depend on the choice of $F \in p^{-1}(H)$. For $p(F)=p(G)$ and $F \neq G$ if and only if $F$ and $G$ have finite domains and $F(k)=H(k)=G(k)$ and for all integers $k$ in their domains. Clearly, $h$ is a roughly isometric map with with constant $a=1$. Moreover, $j$ shows that $h$ is a rough isometry between $T$ and $S$. 
The following definition is well-known. The name "doubling" comes from the fact that usually $C=2$. The version given here appears in Bonk and Schramm [1, Section 9].

Definition 5.8. A metric space is doubling if for every $C>1$ there exists $N \in \mathbf{N}$ such that: if $0<r<R$ with $R / r=C$, then every open ball of radius $R$ in $X$ can be covered by $N$ open balls of radius $r$.

Definition 5.9. A metric space is pseudo-doubling if for every $C>1$ there exists $N \in \mathbf{N}$ such that: if $0<r<R$ with $R / r=C$ and $x \in X$, then there are at most $N$ balls $B$ such that $B(x, r) \subseteq B \subseteq B(x, R)$.

Remark 5.10. The following facts are easy to verify.

(1) Every doubling ultrametric space is pseudo-doubling.

(2) Let $X=\operatorname{end}(T, v)$, where $(T, v)$ is a rooted, geodesically complete, simplicial $\mathbf{R}$-tree. Then $X$ is pseudo-doubling. However, if the set of valences of the vertices of $T$ is unbounded, then $X$ is not doubling.

(3) The closed unit interval $[0,1]$ with its standard metric is a doubling metric space that is not pseudo-doubling.

Corollary 5.11. If $f: X \rightarrow Y$ is a bounded distortion equivalence between bounded, complete, uniformly perfect, pseudo-doubling ultrametric spaces, then $f$ is a $P Q$-symmetric homeomorphism.

Proof. Let $X=\operatorname{end}\left(T_{X}, v\right), Y=\operatorname{end}\left(T_{Y}, w\right)$, where $T_{X}, T_{Y}$ are geodesically complete, bushy R-trees (see Remark 5.3). Since $X$ is pseudo-doubling, for $R / r=\mathrm{e}$ there exists a constant $N$ such that for any $x \in X$ there are at most $N$ balls $B$ such that $B(x, r) \subseteq B \subseteq B(x, R)$. It follows that for each $k \in \mathbf{N}$ there are at most $N$ different real numbers $\alpha_{1}, \ldots, \alpha_{n}$ with $k<\alpha_{1}<\alpha_{2}<\cdots<\alpha_{n}<k+1$ such that whenever $F, G \in \operatorname{end}\left(T_{X}, v\right)$ and $k<(F \mid G)_{v}<k+1$, then $(F \mid G)_{v}=\alpha_{i}$ for some $i=1, \ldots, n$.

We will now distort the tree $T_{X}$ up to rooted homeomorphism so that Remark 4.5 applies. Divide each interval $[k, k+1]$ into $N+1$ subintervals of length $1 /(N+1)$. For any pair of points $F, G \in \operatorname{end}\left(T_{X}, v\right)$, if $(F \mid G)_{v}=\alpha_{i}$, change it to $(F \mid G)_{v}=$ $k+i /(N+1)$. Denote this new tree by $\left[T_{X}\right]$. It is immediate to see that the natural rooted homeomorphism $T_{X} \rightarrow\left[T_{X}\right]$ induces a bi-Lipschitz equivalence end $\left(T_{X}, v\right) \rightarrow$ end $\left(\left[T_{X}\right], v\right)$ with constant e.

Now consider $S_{X}$, a barycentric subdivision of $\left[T_{X}\right]$ obtained by dividing each edge into $N+1$ subintervals of length $1 /(N+1)$ and adding the corresponding $N$ new vertices. Consider each new edge to be of length 1 . Then $S_{X}$ is similar to $\left[T_{X}\right]$ (the distance is multiplied by $N+1$ ) and, by construction of $\left[T_{X}\right], S_{X}$ is simplicial. Also, the canonical map $h_{X}: \operatorname{end}\left(T_{X}, v\right) \rightarrow \operatorname{end}\left(S_{X}, v\right)$ is such that $\left(\frac{1}{\mathrm{e}} d(F, G)\right)^{N+1} \leq$ $d\left(h_{X}(F), h_{X}(Y)\right) \leq(\mathrm{e} \cdot d(F, G))^{N+1}$. Thus, $h_{X}$ is PQ-symmetric.

Likewise distort $T_{Y}$ to $\left[T_{Y}\right]$ and construct $S_{Y}$ and $h_{Y}: \operatorname{end}\left(T_{Y}, w\right) \rightarrow \operatorname{end}\left(S_{Y}, w\right)$.

It follows that $f$ induces a bounded distortion equivalence

$$
\tilde{f}:=h_{Y} \circ f \circ h_{X}^{-1}: \operatorname{end}\left(S_{X}, v\right) \rightarrow \operatorname{end}\left(S_{Y}, w\right)
$$

between ends of rooted, geodesically complete, simplicial, bushy $\mathbf{R}$-trees. Theorem 5.5 implies that $\tilde{f}: \operatorname{end}\left(S_{X}, v\right) \rightarrow \operatorname{end}\left(S_{Y}, w\right)$ is PQ-symmetric. Since $f=$ $h_{Y}^{-1} \circ \tilde{f} \circ h_{X}$, Remark 3.5 implies that $f$ is PQ-symmetric. 


\section{The examples}

This section contains several examples that illustrate the sharpness of the results in the previous sections. In addition, some of the examples answer several questions raised by Mirani $[11,12]$. The basic building block for our examples is the infinite binary tree. We begin by introducing notation that will help us describe the examples.

Notation 6.1. Let $\left(T_{2}, v\right)$ denote the rooted, infinite binary tree, also known as the Cantor tree. Thus, $T_{2}$ is a locally finite, simplicial $\mathbf{R}$-tree, the root has valency two, and all other roots have valency three. All edges are labeled 0 or 1 ; every vertex is incident to at least one edge labeled 0 and to at least one edge labeled 1 . Let $X_{2}=\operatorname{end}(T, v)$. Thus,

$$
X_{2}=\left\{x=\left(x_{1}, x_{2}, \ldots\right) \mid x_{i} \in\{0,1\}, i=1,2, \ldots\right\}=\prod_{1}^{\infty}\{0,1\}
$$

with Gromov product at infinity given by $(x \mid y)_{v}=i$ if and only if $x_{j}=y_{j}$ for $j \leq i$ and $x_{i+1} \neq y_{i+1}$.

If $a=\left(a_{1}, \ldots, a_{n}\right) \in \prod_{1}^{n}\{0,1\}$, then

$$
a X_{2}:=\left\{y \in X_{2} \mid y_{i}=a_{i} \text { for } 1 \leq i \leq n\right\}
$$

Thus, each $y \in a X_{2}$ can be written uniquely as $y=a x$, where $x \in X_{2}$. Note that $a X_{2}$ is a closed ball in $X_{2}$.

We denote certain infinite and finite sequences as follows:

$$
\begin{array}{rlrl}
\overline{0} & =(0,0,0, \ldots) \in X_{2}, & \overline{1} & =(1,1,1, \ldots) \in X_{2}, \\
\overline{0}_{n} & =(0, \ldots, 0) \in \prod_{1}^{n}\{0,1\}, & \overline{1}_{n}=(1, \ldots, 1) \in \prod_{1}^{n}\{0,1\} .
\end{array}
$$

If $n=0$, then $\overline{0}_{n}$ and $\overline{1}_{n}$ denote the empty sequences.

Since much of Ghys and de la Harpe [5] and Mirani [11, 12] is in the setting of simplicial $\mathbf{R}$-trees with the property that each vertex has valency at least 3 , we want to provide examples in that class of trees whenever possible. A simple way to achieve this is to let $\left.\left(T_{3}, v\right)\right)$ be the unique rooted, simplicial $\mathbf{R}$-tree containing $\left(T_{2}, v\right)$ as a rooted subtree such that every vertex of $T_{3}$ has valency exactly 3 . In other words, $T_{3}$ is the infinite 3 -regular tree.

Let $X_{3}=\operatorname{end}\left(T_{3}, v\right)$. Note that $X_{2}$ and $X_{3}$ are compact, uniformly perfect, doubling ultrametric spaces. Moreover, $X_{2}$ is a closed and open subspace of $X_{3}$.

The first example shows that the hypothesis of Theorems 1.1 and 5.5 can not be changed from "bounded distortion equivalence" to "bi-Hölder and conformal homeomorphism".

Example 6.2. There exist a bi-Hölder, conformal homeomorphism $h: X_{3} \rightarrow X_{3}$ that does not have bounded distortion. In particular, $h$ is not $P Q$-symmetric. 
Proof. For each $i \geq 1$, define the following sequences:

$$
\begin{aligned}
a_{i} & =\overline{1}_{i-1} 011, \\
b_{i} & =\overline{1}_{i-1} \overline{0}_{i+1} 11, \\
c_{i} & =\overline{1}_{i-1} 010, \\
F_{i} & =\overline{1}_{i-1} \overline{0} .
\end{aligned}
$$

Define a homeomorphism $h: X_{3} \rightarrow X_{3}$ by

$$
\begin{cases}h\left(a_{i} x\right)=b_{i} x & \text { for all } x \in X_{2} \text { and } i=1,2, \ldots, \\ h\left(b_{i} x\right)=a_{i} x & \text { for all } x \in X_{2} \text { and } i=1,2, \ldots, \\ h(x)=x & \text { for all } x \in X_{3} \backslash \bigcup_{i=1}^{\infty}\left(a_{i} X_{2} \cup b_{i} X_{2}\right) .\end{cases}
$$

To see that $h$ is conformal, note that if $\overline{1} \neq F \in X_{2}$, then there exists $n_{F} \geq 1$ such that the $n_{F}{ }^{\text {th }}$ term of $F$ is 0 . It follows that the image under $h$ of any sphere centered at $F$ of radius $r \leq \mathrm{e}^{-1-n_{F}}$ is a sphere; thus, $D_{h}(F, r)=1$. Clearly, for all $r>0$, $D_{h}(\overline{1}, r)=1$ and $\bar{D}_{h}(F, r)=1$ whenever $F \in X_{3} \backslash X_{2}$. Thus, $h$ is conformal.

To see that $h$ is bi-Hölder, it suffices to check that for any pair of points $F, G \in X_{3}$, $\frac{1}{2}(F \mid G)_{v}-1 \leq(h(F) \mid h(G))_{v} \leq 2(F \mid G)_{v}+1$. It only happens that $(h(F) \mid h(G))_{v} \neq$ $(F \mid G)_{v}$ when

(1) one of $F$ or $G$ is in $a_{i} X_{2}$ or $b_{i} X_{2}$ and the other is in $X_{3} \backslash \bigcup_{i=1}^{\infty}\left(a_{i} X_{2} \cup b_{i} X_{2}\right)$, or

(2) $F, G \in a_{i} X_{2}$ for some $i \geq 1$, or

(3) $F, G \in b_{i} X_{2}$ for some $i \geq 1$.

In each case, the required inequalities are easy to check.

To see that $h$ does not have bounded distortion, choose $G_{i} \in a_{i} X_{2}$ and $H_{i} \in c_{i} X_{2}$ for each $i \geq 1$. Note that $h\left(F_{i}\right)=F_{i}, h\left(G_{i}\right) \in b_{i} X_{2}$, and $h\left(H_{i}\right)=H_{i}$ for all $i \geq 1$. Moreover, $\left(F_{i} \mid G_{i}\right)_{v}=\left(F_{i} \mid H_{i}\right)_{v}=i$ and $\left(h\left(F_{i}\right) \mid h\left(G_{i}\right)\right)_{v}=2 i$ for all $i \geq 1$. Thus, $\mid\left(h\left(F_{i} \mid h\left(G_{i}\right)_{v}-\left(h\left(F_{i} \mid h\left(H_{i}\right)\right)_{v} \mid=i\right.\right.\right.$ and Proposition 4.1 shows that $h$ does not have bounded distortion.

Finally, it follows from Proposition 3.6 that $h$ is not PQ-symmetric.

The following example illustrates that the result of Tukia and Väisälä [17, Theorem 3.14] mentioned in Remark 3.8 above, that a PQ-symmetric homeomorphism between bounded metric spaces is bi-Hölder, does not hold if the hypothesis "PQsymmetric" is weakened to "quasiconformal", even for ultrametric spaces as nice as $X_{3}$.

Example 6.3. There exists a conformal homeomorphism $h: X_{3} \rightarrow X_{3}$ that is not bi-Hölder.

Proof. For each $i \geq 1$, define the following sequences:

$$
\begin{aligned}
g_{i} & =\overline{1}_{i-1} 01, \\
h_{i} & =\overline{1}_{i-1} \overline{0}_{i^{2}-i} 1 .
\end{aligned}
$$

Define a homeomorphism $h: X_{3} \rightarrow X_{3}$ by

$$
\begin{cases}h\left(g_{i} x\right)=h_{i} x & \text { for all } x \in X_{2} \text { and } i=1,2, \ldots, \\ h\left(h_{i} x\right)=g_{i} x & \text { for all } x \in X_{2} \text { and } i=1,2, \ldots, \\ h(x)=x & \text { for all } x \in X_{3} \backslash \bigcup_{i=1}^{\infty}\left(g_{i} X_{2} \cup h_{i} X_{2}\right) .\end{cases}
$$


The argument to prove that $h$ is conformal is similar to the one in Example 6.2. To see that $h$ is not bi-Hölder, for each $i=1,2,3, \ldots$ choose $G_{i} \in g_{i} X_{2}$ and let $F_{i} \in X_{2}$ be as in Example 6.2. Note that $h\left(F_{i}\right)=F_{i}$ and $h\left(G_{i}\right) \in h_{i} X_{2}$ for all $i \geq 1$. Thus, $d\left(h\left(F_{i}\right), h\left(G_{i}\right)\right)=\mathrm{e}^{-i^{2}}$ while $d\left(F_{i}, G_{i}\right)=\mathrm{e}^{-i}$ for all $i \geq 1$. It follows that $h$ is not bi-Hölder.

In the converse direction, Mirani $[11,12]$ speculated that bi-Hölder homeomorphisms on spaces such as $X_{3}$ might be quasi-conformal. The following example shows this is not the case.

Example 6.4. There exists a bi-Hölder homeomorphism $h: X_{3} \rightarrow X_{3}$ that is not not quasiconformal.

Proof. For each $i \geq 1$, define the following sequences:

$$
\begin{aligned}
g_{i} & =\overline{0}_{4^{i}} 11, \\
h_{i} & =\overline{0}_{2 \cdot 4^{i}} 11 .
\end{aligned}
$$

Define a homeomorphism $h: X_{3} \rightarrow X_{3}$ by

$$
\begin{cases}h\left(g_{i} x\right)=h_{i} x & \text { for all } x \in X_{2} \text { and } i=1,2, \ldots, \\ h\left(h_{i} x\right)=g_{i} x & \text { for all } x \in X_{2} \text { and } i=1,2, \ldots, \\ h(x)=x & \text { for all } x \in X_{3} \backslash \bigcup_{i=1}^{\infty}\left(g_{i} X_{2} \cup h_{i} X_{2}\right) .\end{cases}
$$

The argument to prove that $h$ is bi-Hölder is similar to the one in Example 6.2: one checks that $F, G \in \operatorname{end}(T, v), \frac{1}{2}(F \mid G)_{v}-1 \leq(h(F) \mid h(G))_{v} \leq 2(F \mid G)_{v}+1$ for every $F, G \in X_{3}$.

To see that $h$ is not quasiconformal, it suffices to show that $\limsup _{\epsilon \rightarrow 0} D_{h}(\overline{0}, \epsilon)=\infty$. To this end, let $K>0$ and $N \in \mathbf{N}$ be given. There exists $i_{0}>N$ such $4^{i_{0}}>K$. Choose $G_{1} \in g_{i_{0}} X_{2}$ and $G_{2} \in \overline{0}_{4^{i_{0}}} 10 X_{2}$. It follows that $h(\overline{0})=\overline{0}, h\left(G_{1}\right) \in h_{i_{0}} X_{2}$, and $h\left(G_{2}\right)=G_{2}$. Therefore, $\left(h\left(F_{0}\right) \mid h\left(G_{2}\right)\right)_{v}=4^{i_{0}}$ and $\left(h\left(F_{0}\right) \mid h\left(G_{1}\right)\right)_{v}=2 \cdot 4^{i_{0}}$. Hence, $D_{h}\left(\overline{0}, \mathrm{e}^{-4^{i_{0}}}\right) \geq \frac{\mathrm{e}^{-4^{i} 0}}{\mathrm{e}^{-2 \cdot 4^{i} 0}}=\mathrm{e}^{4^{i_{0}}}$.

For functorial reasons, Mirani [11, 12] was interested in compositions of bi-Hölder, quasiconformal homeomorphisms between spaces such as $X_{3}$. The following example illustrates that these compositions need not be well-behaved. This is in contrast to the situation for PQ-symmetric homeomorphisms as observed by Tukia and Väisälä [17, Theorem 2.2] (see Remark 3.5).

Example 6.5. There exist two bi-Hölder, quasiconformal homeomorphisms

$$
h_{1}, h_{2}: X_{3} \rightarrow X_{3}
$$

such that the composition $h_{2} \circ h_{1}$ is not quasiconformal. Moreover, $h_{1}$ and $h_{2}$ are bounded distortion equivalences and $h_{2}$ is conformal.

Proof. For each $i \geq 1$, define the following sequences:

$$
\begin{aligned}
g_{i}^{a} & =\overline{0}_{2 i} 11, \\
h_{i}^{a} & =\overline{0}_{2 i+1} 11, \\
g_{i}^{b} & =\overline{0}_{4 i} 1, \\
h_{i}^{b} & =\overline{0}_{8 i} 1 .
\end{aligned}
$$


Define a homeomorphism $h_{1}: X_{3} \rightarrow X_{3}$ by

$$
\begin{cases}h_{1}\left(g_{i}^{a} x\right)=h_{i}^{a} x & \text { for all } x \in X_{2} \text { and } i=1,2, \ldots, \\ h_{1}\left(h_{i}^{a} x\right)=g_{i}^{a} x & \text { for all } x \in X_{2} \text { and } i=1,2, \ldots, \\ h_{1}(x)=x & \text { for all } x \in X_{3} \backslash \bigcup_{i=1}^{\infty}\left(g_{i}^{a} X_{2} \cup h_{i}^{a} X_{2}\right) .\end{cases}
$$

Define a homeomorphism $h_{2}: X_{3} \rightarrow X_{3}$ by

$$
\begin{cases}h_{2}\left(g_{i}^{b} x\right)=h_{i}^{b} x & \text { for all } x \in X_{2} \text { and } i=1,2, \ldots, \\ h_{2}\left(h_{i}^{b} x\right)=g_{i}^{b} x & \text { for all } x \in X_{2} \text { and } i=1,2, \ldots, \\ h_{2}(x)=x & \text { for all } x \in X_{3} \backslash \bigcup_{i=1}^{\infty}\left(g_{i}^{b} X_{2} \cup h_{i}^{b} X_{2}\right) .\end{cases}
$$

It is readily seen that $h_{1}$ is e-quasiconformal. Indeed, $d_{h_{1}}(F, \epsilon) \leq$ e for all $F \in X_{3}$ and for all $\epsilon>0$, from which it also follows that $h_{1}$ is a bounded distortion equivalence. Likewise, $d_{h_{2}}(F, \epsilon)=1$ for all $F \in X_{3}$ and for all $\epsilon>0$, from which it also follows that $h_{2}$ is conformal and a bounded distortion equivalence.

The next two examples show that "uniformly perfect" can not be weakened to "perfect" in Theorem 1.1 and Corollary 5.11, and that the "bushy" condition is needed in Theorem 5.5.

Example 6.6. There exist a compact, perfect, pseudo-doubling, ultrametric space $Z$ and a conformal homeomorphism $h: Z \rightarrow Z$ such that $h$ is a bounded distortion equivalence, but $h$ is not quasi-symmetric. Moreover, $h$ can be chosen to be bi-Hölder or not bi-Hölder.

Proof. We begin by constructing compact, perfect, pseudo-doubling, ultrametric spaces $X$ and $Y$ and a conformal, bounded distortion equivalence $f: X \rightarrow Y$ such that $f$ is neither quasi-symmetric nor bi-Hölder. Let $\left(T_{2}^{i}, v_{i}\right)$ denote a copy of the rooted, infinite binary tree $(T, v)$ for each $i \geq 1$. Form the tree $T=T_{2} \bigvee_{i=1}^{\infty} T_{2}^{i}$ by attaching, for each $i \geq 1, T_{2}^{i}$ to $T_{2}$ by identifying $v_{i}$ with the vertex on $\overline{1} \subseteq T_{2}$ that is a distance $i$ from $v$. Note that $(T, v)$ is a rooted, geodesically complete, simplicial $\mathbf{R}$-tree.

For each $i \geq 1$, let $X_{2}^{i}=\operatorname{end}\left(T_{2}^{i}, v_{i}\right)$. Then

$$
X:=\operatorname{end}(T, v)=X_{2} \bigcup_{i=1}^{\infty} \overline{1}_{i} X_{2}^{i}
$$

As before, the points of $X_{2}$ are infinite sequences of 0 's and 1's. For each $i \geq 1$, the points of $X_{2}^{i}$ are denoted by infinite sequences of $0_{i}$ 's and $1_{i}$ 's. A typical point of $X$ is either a point of $X_{2}$ or of the form $\overline{1}_{i} x=(\underbrace{1, \ldots, 1}_{i}) x$, where $x \in X_{2}^{i}$. Note that $X$ is a compact, uniformly perfect, pseudo-doubling, ultrametric space.

For each $i \geq 1$ define

$Y_{2}^{i}=\left\{y \in X_{2}^{i} \mid y=(\underbrace{x_{1}, \ldots, x_{1}}_{i}, \underbrace{x_{2}, \ldots, x_{2}}_{i}, \underbrace{x_{3}, \ldots, x_{3}}_{i}, \ldots), x_{j} \in\left\{0_{i}, 1_{i}\right\}\right.$ for each $\left.j \geq 1\right\}$.

Note that for each $i \geq 1, Y_{2}^{i}$ is a closed, uniformly perfect subset of $X_{2}^{i}$. There is an evident subtree $S_{2}^{i}$ of $T_{2}^{i}$ such that end $\left(S_{2}^{i}, v_{i}\right)=Y_{2}^{i}$. The vertices of $S_{2}^{i}$ of valency 3 are those vertices of $T_{2}^{i}$ that are a distance $n i$ from $v_{i}$ for some $n=1,2,3, \ldots$ 
Let $T^{\prime}=T_{2} \bigvee_{i=1}^{\infty} S_{2}^{i}$ so that $\left(T^{\prime}, v\right)$ is a rooted, geodesically complete subtree of $(T, v)$. For each $i \geq 1$, let $Y_{2}^{i}=\operatorname{end}\left(S_{2}^{i}, v_{i}\right)$. Then

$$
Y:=\operatorname{end}\left(T^{\prime}, v\right)=X_{2} \bigcup_{i=1}^{\infty} \overline{1}_{i} Y_{2}^{i}
$$

Note that $Y$ is a compact, perfect, pseudo-doubling, ultrametric space. The simplicial $\mathbf{R}$-tree $T^{\prime}$ is not bushy because for any $K>0$, if $i \geq 2 K$, then there are points $x$ in $S_{2}^{i}$ such that if $y$ is within $K$ of $x$, then $T^{\prime} \backslash\{y\}$ has exactly 2 components. In particular, $Y$ is not uniformly perfect.

Define $f: X \rightarrow Y$ by

$$
f\left(\overline{1}_{i} x\right)= \begin{cases}x & \text { if } i=0 \text { and } x \in X_{2}, \\ \overline{1}_{i}(\underbrace{x_{1}, \ldots, x_{1}}_{i}, \underbrace{x_{2}, \ldots, x_{2}}_{i}, \underbrace{x_{3}, \ldots, x_{3}}_{i}, \ldots) & \text { if } i \geq 1 \text { and } x \in X_{2}^{i} .\end{cases}
$$

Note that $f\left(X_{2}\right)=Y_{2}$ and $f\left(X_{2}^{i}\right)=Y_{2}^{i}$ for all $i \geq 1$.

Clearly, $f$ is induced by a rooted homeomorphism $\hat{f}:(T, v) \rightarrow\left(T^{\prime}, v\right)$; i.e., $f(F)=$ $\hat{f} \circ F$ for each $F \in \operatorname{end}(T, v)$. It follows from Remark 4.5 that $f$ is conformal and $f$ is a bounded distortion equivalence.

To see that $f$ is not quasi-symmetric, choose $G \in X_{2}$ and $F_{i}, H_{i} \in X_{2}^{i}$ such that $\left(F_{i} \mid H_{i}\right)_{v}=i+1$ for each $i \geq 1$. Then $\left|\left(F_{i} \mid H_{i}\right)_{v}-\left(F_{i} \mid G\right)_{v}\right|=1$ and

$$
\left|\left(f\left(F_{i}\right) \mid f\left(H_{i}\right)\right)_{v}-\left(f\left(F_{i}\right) \mid f(G)\right)_{v}\right|=i
$$

for each $i \geq 1$. Proposition 4.2 implies that $f$ is not quasi-symmetric.

To verify that $f$ is not bi-Hölder, choose for each $i \geq 1, F_{i}, G_{i} \in X_{2}^{i}$ such that $\left(F_{i} \mid G_{i}\right)_{v}=2 i$. Then $\left(f\left(F_{i}\right) \mid f\left(G_{i}\right)\right)_{v}=i+i^{2}$. It follows that the criterion in Remark 4.4 is violated.

We have now constructed a conformal, bounded distortion equivalence $f: X \rightarrow Y$ that is neither quasi-symmetric nor bi-Hölder. In order, to get an example in which the domain and range are the same, let $Z=\operatorname{end}\left(T \vee T^{\prime}, v\right)$ so that $Z=X \cup Y$ and define $h: Z \rightarrow Z$ by $h_{X}=f$ and $h \mid Y=f^{-1}$.

Finally, we briefly indicate the modifications that need to be made in order to get a bi-Hölder example. For each $i \geq 1$ replace $Y_{2}^{i}$ by

$$
\hat{Y}_{2}^{i}=\left\{y \in X_{2}^{i} \mid y=(\underbrace{x_{1}, \ldots, x_{1}}_{i}, x_{2}, x_{3}, \ldots), x_{j} \in\left\{0_{i}, 1_{i}\right\} \text { for each } j \geq 1\right\}
$$

and replace $f$ by

$$
\hat{f}\left(\overline{1}_{i} x\right)= \begin{cases}x & \text { if } i=0 \text { and } x \in X_{2}, \\ \overline{1}_{i}(\underbrace{x_{1}, \ldots, x_{1}}_{i}, x_{2}, x_{3}, \ldots) & \text { if } i \geq 1 \text { and } x \in X_{2}^{i}\end{cases}
$$

These changes are enough to construct a bi-Hölder, conformal, bounded distortion equivalence that is not quasi-symmetric.

Example 6.7. There exist a compact, perfect, pseudo-doubling, ultrametric space $Z$ and a conformal homeomorphism $h: Z \rightarrow Z$ such that $h$ is quasi-symmetric, but $h$ is not PQ-symmetric. Moreover, $h$ can be chosen to be bi-Hölder or not biHölder. 
Proof. These examples are modifications of Example 6.6. Therefore, we will just briefly indicate the changes that need to be made.

We begin by showing how to produce the example that is not bi-Hölder. Let $Y$ be as in Example 6.6. Construct a space $W$ as $Y$ is constructed above except that $Y_{2}^{i}$ is replaced by

$\tilde{Y}_{2}^{i}=\left\{y \in X_{2}^{i} \mid y=(\underbrace{x_{1}, \ldots, x_{1}}_{i^{2}}, \underbrace{x_{2}, \ldots, x_{2}}_{i^{2}}, \underbrace{x_{3}, \ldots, x_{3}}_{i^{2}}, \ldots), x_{j} \in\left\{0_{i}, 1_{i}\right\}\right.$ for each $\left.j \geq 1\right\}$.

Let

$$
\tilde{Y}=X_{2} \bigcup_{i=1}^{\infty} \overline{1}_{i} \tilde{Y}_{2}^{i}
$$

and define $\tilde{f}: Y \rightarrow \tilde{Y}$ by $\tilde{f}(x)=x$ if $x \in X_{2}$ and

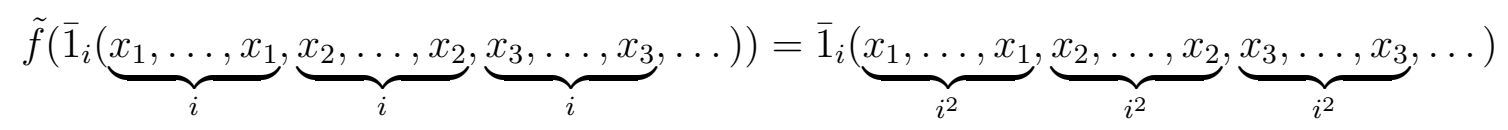

if $i \geq 1$ and $\left(x_{1}, x_{2}, x_{3}, \ldots\right) \in X_{2}^{i}$. It follows from Remark 4.5 that $\tilde{f}$ is conformal. One can verify that $\tilde{f}$ is quasi-symmetric by invoking Proposition 4.2 with

$$
\gamma(t)= \begin{cases}t^{2} & \text { if } t \geq 0 \\ t & \text { if } t<0\end{cases}
$$

To verify that $f$ is not bi-Hölder, choose for each $i \geq 1, F_{i}, G_{i} \in Y_{2}^{i}$ such that $\left(F_{i} \mid G_{i}\right)_{v}=2 i$. Then $\left(\tilde{f}\left(F_{i}\right) \mid \tilde{f}\left(G_{i}\right)\right)_{v}=i+i^{2}$. It follows that the criterion in Remark 4.4 is violated. To see that $\tilde{f}$ is not PQ-symmetric, choose $G \in X_{2}$ and $F_{i}, H_{i} \in Y_{2}^{i}$ such that $\left(F_{i} \mid H_{i}\right)_{v}=2 i$ for each $i \geq 1$. Then $\left|\left(F_{i} \mid H_{i}\right)_{v}-\left(F_{i} \mid G\right)_{v}\right|=i$ and

$$
\left|\left(\tilde{f}\left(F_{i}\right) \mid \tilde{f}\left(H_{i}\right)\right)_{v}-\left(\tilde{f}\left(F_{i}\right) \mid \tilde{f}(G)\right)_{v}\right|=i^{2}
$$

for each $i \geq 1$. Proposition 4.3 implies that $f$ is not PQ-symmetric. The passage from $\tilde{f}: Y \rightarrow \tilde{Y}$ to $h: Z \rightarrow Z$ takes place as in Example 6.6.

To construct the bi-Hölder example, replace $\tilde{f}: Y \rightarrow \tilde{Y}$ by $\tilde{f}^{\prime}: Y^{\prime} \rightarrow \tilde{Y}^{\prime}$ with the following explanations. First, just as $Y$ arises by attaching trees $S_{2}^{i}$ to points on $\overline{1}$ a distance $i$ from $v$, the space $Y^{\prime}$ arises by attaching those trees to points a distance $i^{2}$ from $v$. Second, just as $\tilde{Y}$ arises from a modification of $Y$ by replacing each $Y_{2}^{i}$ by $\tilde{Y}_{2}^{i}$, the space $\tilde{Y}^{\prime}$ arises by replacing $Y_{2}^{i} \subseteq Y^{\prime}$ by

$$
\begin{gathered}
\left(\widetilde{Y}_{2}^{i}\right)^{\prime}=\left\{y \in X_{2}^{i} \mid y=(\underbrace{x_{1}, \ldots, x_{1}}_{i^{2}}, \underbrace{x_{2}, \ldots, x_{2}}_{i}, \underbrace{x_{3}, \ldots, x_{3}}_{i}, \ldots),\right. \\
\left.x_{j} \in\left\{0_{i}, 1_{i}\right\} \text { for each } j \geq 1\right\} .
\end{gathered}
$$

The map $\tilde{f}^{\prime}$ is obtained by modifying $\tilde{f}$ in the obvious way.

The final example shows the necessity of the "pseudo-doubling" in Theorem 1.1 and Corollary 5.11.

Example 6.8. There exist a compact, uniformly perfect, ultrametric space $X$ that is not pseudo-doubling and a bounded distortion equivalence $f: X \rightarrow X_{3}$ that is not $P Q$-symmetric. Moreover, $f$ is not bi-Hölder. 
Proof. For each $i \in \mathbf{N}$ consider the edge $e_{i}$ on the tree $T_{2} \subseteq T_{3}$ joining $\overline{1}_{i}$ to $\overline{1}_{i+1}$. Choose points $\overline{1}_{i}=x_{i}^{0}, x_{i}^{1}, \ldots, x_{i}^{i}, x_{i}^{i+1}=\overline{1}_{i+1}$ dividing $e_{i}$ into $i+1$ subintervals of length $1 /(i+1)$. For each $i \in \mathbf{N}$ and to each point $x_{i}^{j}, 1 \leq j \leq i$, attach a copy $T_{i}^{j}$ of $T_{2} \subseteq T_{3}$ to $T_{3}$ by identifying the root $v_{i}^{j}$ of $T_{i}^{j}$ to $x_{i}^{j}$. Let $T=T_{3} \bigvee_{i=1}^{\infty} \bigvee_{j=1}^{i} T_{i}^{j}$ be the resulting tree and let $X=\operatorname{end}(T, v)$. Define $f: X \rightarrow X_{3}$ as follows. If $x \in\left(X \backslash \operatorname{end}\left(T_{2}, v\right)\right) \cup 0 X_{2}$, define $f(x)=x$. If $x \in 1 X_{2}$ is of the form $x=\overline{1}_{i}$ for some $i \in \mathbf{N}$, define $f(x)=\overline{1}_{i^{2}} y$. Any other point $x \in X$ may be written (using self-explanatory notation) as $x=\overline{1}_{i} x_{i}^{1} \ldots x_{i}^{j} z$, where $i \in \mathbf{N}, 1 \leq j \leq i$, and $z=z_{1} z_{2} z_{3} \ldots \in X_{2}$. For such an $x$, define $f(x)=\overline{1}_{i+2 j+z_{1}-1} z_{2} z_{3} z_{4} \ldots$ It can be verified that $f$ is a bounded distortion equivalence (with constant e) and that $f$ is neither PQ-symmetric nor bi-Hölder.

\section{References}

[1] Bonk, M., and O. Schramm: Embeddings of Gromov hyperbolic spaces. - Geom. Funct. Anal. 10:2, 2000, 266-306.

[2] Bridson, M. R., and A. Haffliger: Metric spaces of non-positive curvature. - Grundlehren Math. Wiss. 319, Springer-Verlag, Berlin, 1999.

[3] Buyalo, S., and Schroeder, V.: Elements of asymptotic geometry. - EMS Monogr. Math., European Mathematical Society (EMS), Zürich, 2007.

[4] Feřnberg, V.Z.: Compact ultrametric spaces. - Dokl. Akad. Nauk SSSR 214, 1974, 10411044.

[5] Ghys, É., and P. DE LA Harpe: Sur les groupes hyperboliques d'après Mikhael Gromov. Progr. Math. 83, Birkhäuser Boston Inc., Boston, MA, 1990.

[6] Heinonen, J.: Lectures on analysis on metric spaces. - Universitext, Springer-Verlag, New York, 2001.

[7] Hughes, B.: Trees and ultrametric spaces: a categorical equivalence. - Adv. Math. 189:1, 2004, 148-191.

[8] Hughes, B.: Trees, ultrametrics, and noncommutative geometry. - Pure Appl. Math. Q. (to appear).

[9] Martínez-Pérez, Á.: Quasi-isometries between visual hyperbolic spaces. - Preprint, arXiv:0810.4505.

[10] Martínez-Pérez, Á., and M. A. Morón: Uniformly continuous maps between ends of Rtrees. - Math. Z. 263:3, 2009, 583-606.

[11] Mirani, M.: Classical trees and compact ultrametric spaces. - Dissertation, Vanderbilt University, 2006.

[12] Mirani, M.: Classical trees and compact ultrametric spaces. - Preprint, 2008.

[13] Mosher, L., M. Sageev, and K. Whyte: Quasi-actions on trees. I. Bounded valence. - Ann. of Math. (2) 158:1, 2003, 115-164.

[14] Paulin, F.: Un groupe hyperbolique est déterminé par son bord. - J. London Math. Soc. (2) $54: 1,1996,50-74$.

[15] Roe, J.: Lectures on coarse geometry. - Univ. Lecture Ser. 31, AMS, Providence, RI, 2003.

[16] Semmes, S.: Metric spaces and mappings seen at many scales. - Appendix in: Metric Structures for Riemannian and Non-Riemannian Spaces (by M. Gromov et al.), Birkhäuser, 1999.

[17] Tukia, P., and J. VÄısÄLÄ: Quasisymmetric embeddings of metric spaces. - Ann. Acad. Sci. Fenn. Ser. A I Math. 5:1, 1980, 97-114.

Received 1 September 2009 